\title{
ON THE ASYMPTOTICS OF THE TRAPEZOIDAL RULE FOR THE PANTOGRAPH EQUATION
}

\author{
J. ČERMÁK AND J. JÁNSKÝ
}

\begin{abstract}
The paper deals with the trapezoidal rule discretization of a class of linear delay differential equations, with a special emphasis on equations with a proportional delay. Our purpose is to analyse the asymptotic properties of the numerical solutions and formulate their upper bounds. We also survey the known results and show that our formulae improve and generalize these results. In particular, we set up conditions under which the numerical solution of the scalar pantograph equation has the same decay rate as the exact solution.
\end{abstract}

\section{INTRODUCTION}

In recent years, many papers have been focused on the analysis of the exact and numerical solution of differential equations involving proportional delays. The prototype of such equations is the pantograph equation

$$
y^{\prime}(t)=a y(t)+b y(\lambda t), \quad t \geq 0,
$$

where $a, b \neq 0$ are (generally complex) scalars and $0<\lambda<1$ is a real scalar. The equation (1.1) and its modifications (involving especially the vector case and the neutral case) have appeared as a mathematical idealization of many problems in various areas comprising number theory, astrophysics or electrodynamics (see, e.g., [17] and the references cited therein).

Nevertheless, the broad applicability of these equations is not the only (nor probably the main) reason why their investigation has attracted the attention of numerous mathematicians. The typical features of the qualitative as well as numerical analysis of these equations are namely very specific and sometimes also surprising properties of their solutions. The crucial results concerning the stability and asymptotics of the exact solutions are involved in papers [11, 13, 14, 17, 18, 22. The different techniques ranging from the approach of Dirichlet series to the variationof-constants method have been successfully employed in these papers to analyse the correct behavior of the exact solutions on the unbounded interval.

Since the last decade, a very intensive investigation of numerical methods for various families of pantograph equations has been started. The frequent topics of this analysis are numerical methods based on the Taylor polynomials as well as the discussions on the stability of special classes of Runge-Kutta methods (see,

Received by the editor December 18, 2007, and, in revised form, November 15, 2008.

2000 Mathematics Subject Classification. Primary 34K28, 39A11; Secondary 65L05, 65L20.

Key words and phrases. Pantograph equation, asymptotic behavior, trapezoidal rule.

The authors were supported by the research plan MSM 0021630518 "Simulation modelling of mechatronic systems" of the Ministry of Education, Youth and Sports of the Czech Republic and by the grant \# 201/08/0469 of the Czech Grant Agency. 
e.g., [9, 15, 21, 23]). In particular, special attention was paid to the trapezoidal rule representing the favourite starting point to the analysis of standard numerical methods. The discussions on this type of discretization resulted in a series of papers 3. 10, 12, 20] describing its stability and asymptotic properties as well as some problems on the compact domain. A general reference for the numerical analysis of the pantograph equation and other delay equations is the book 1 .

The purpose of this paper is to study the asymptotic behavior of the trapezoidal rule applied to the nonautonomous pantograph equation

$$
y^{\prime}(t)=a(t) y(t)+b(t) y(\lambda t), \quad t \geq 0,
$$

where $0<\lambda<1$ is a real scalar and $a, b$ are nonzero continuous complex-valued functions on $[0, \infty)$. In Section 2, we briefly survey essentials on the trapezoidal rule for (1.1) and (1.2) including the references to some relevant results. Section 3 presents the description of the asymptotic properties of this discretization. The main result of this section formulates the upper bound for the numerical solution of (1.2) in terms of a solution of an auxiliary difference inequality. This asymptotic result is applied to the numerical discretization of (1.1) and compared with the known upper bounds for the numerical and exact solution of (1.1). This comparison shows that the trapezoidal rule discretization of (1.1) with real scalars $a, b$ retains the same algebraic bounds as possessed by the exact solution. Some numerical examples illustrating these results are given as well. In the final Section 4, some possible extensions to the equation (1.2) involving a general (not specified) delayed argument are discussed. The procedure employed in this generalization significantly utilizes the Schröder equation. This functional equation and its solution appear also in the main asymptotic result of this section generalizing the upper bound for the numerical solution of the pantograph equation to the case of a related class of equations with a general delay. To illustrate our asymptotic result we present some numerical investigations of the differential equation with a power delayed argument.

\section{SOME PRELIMINARIES}

We consider the discretization of the equation (1.2) by use of the trapezoidal rule in the form

$$
y_{n+1}=R_{n} y_{n}+S_{n} h\left(\beta_{n} y_{\lfloor\lambda n\rfloor}+\alpha_{n} y_{\lfloor\lambda n\rfloor+1}\right), \quad n=0,1, \ldots,
$$

where $y_{n} \approx y(n h), h$ is the stepsize,

$$
R_{n}:=\frac{2+h a_{n}}{2-h a_{n+1}}, \quad S_{n}:=\frac{2 b_{n}}{2-h a_{n+1}}
$$

with $a_{n}:=a(n h), b_{n}:=b(n h)$ and

$$
\alpha_{n}:=\left(\lambda n-\lfloor\lambda n\rfloor+\frac{b_{n+1}}{b_{n}}(\lambda n+\lambda-\lfloor\lambda n\rfloor)\right) / 2, \quad \beta_{n}:=\left(1+\frac{b_{n+1}}{b_{n}}\right) / 2-\alpha_{n} .
$$

The scheme (2.1) is proposed via the integration of (1.2) and the subsequent numerical discretization of both integral terms. While the replacement of the first integral is quite standard, the utilization of a trapezoidal rule approximation of the second integral requires some calculations, particularly a piecewise-linear interpolation of the delayed term $y(\lambda t)$ on the given grid. A more detailed description of this procedure can be found in 3$]$. 
Similarly we can discretize the equation (1.2) involving a general delayed argument, i.e. the equation

$$
y^{\prime}(t)=a(t) y(t)+b(t) y(\theta(t)), \quad t \geq t_{0},
$$

where $\theta$ is a differentiable function which is strictly monotonically increasing and satisfies $\theta\left(t_{0}\right)=t_{0}, \theta(t)<t$ for all $t>t_{0}$. If we set $\psi(t):=\theta^{-1}(t), \theta_{n}:=\theta\left(t_{0}+n h\right)$, $\bar{\theta}_{n}:=\left(\theta_{n}-t_{0}\right) / h, a_{n}:=a\left(t_{0}+n h\right)$ and $b_{n}:=b\left(t_{0}+n h\right)$, where $h$ is the stepsize, then the above sketched procedure yields the recurrence relation

$$
y_{n+1}=R_{n} y_{n}+S_{n} h\left(\beta_{n} y_{\left\lfloor\bar{\theta}_{n}\right\rfloor}+\alpha_{n} y_{\left\lfloor\bar{\theta}_{n}\right\rfloor+1}\right),
$$

where $y_{n} \approx y\left(t_{0}+n h\right), R_{n}, S_{n}$ are given by (2.2) and

$$
\begin{gathered}
\alpha_{n}=\frac{1}{2 h}\left(\theta_{n+1}-\theta_{n}\right)\left(\psi^{\prime}\left(\theta_{n}\right)\left(\bar{\theta}_{n}-\left\lfloor\bar{\theta}_{n}\right\rfloor\right)+\frac{b_{n+1}}{b_{n}} \psi^{\prime}\left(\theta_{n+1}\right)\left(\bar{\theta}_{n+1}-\left\lfloor\bar{\theta}_{n}\right\rfloor\right)\right), \\
\beta_{n}=\frac{1}{2 h}\left(\theta_{n+1}-\theta_{n}\right)\left(\psi^{\prime}\left(\theta_{n}\right)+\frac{b_{n+1}}{b_{n}} \psi^{\prime}\left(\theta_{n+1}\right)\right)-\alpha_{n} .
\end{gathered}
$$

The asymptotic investigation of equations (2.3) and (2.4) is less developed than the study of their particular cases (1.2) and (2.1). Among those papers related to our discussions on (2.3) we refer to the papers [8, 22, 5], where some asymptotic estimations for the equation (2.3) with infinite time lag (i.e. such that lim $\sup (t-$ $\theta(t))=\infty$ as $t \rightarrow \infty$ ) have been performed. The derivation of the corresponding trapezoidal rule discretization (2.4) as well as discussions on the stability analysis of (2.4) belong to the topics of the papers [3, 10].

If we consider the autonomous case (1.1), then the corresponding discretization (2.1) can be simplified as

$$
y_{n+1}=R y_{n}+S h\left(\beta_{n} y_{\lfloor\lambda n\rfloor}+\alpha_{n} y_{\lfloor\lambda n\rfloor+1}\right),
$$

where

$$
R=\frac{2+h a}{2-h a}, \quad S=\frac{2 b}{2-h a}, \quad \alpha_{n}=\lambda n-\lfloor\lambda n\rfloor+\frac{\lambda}{2}>0, \quad \beta_{n}=1-\alpha_{n} .
$$

The formula (2.5) has been exhaustively analysed on the compact as well as unbounded domain. In particular, a very curious property of (2.5) has been reported in [20]. Assuming that $a, b$ are real scalars, $|a|+b<0$ and $0<1-\lambda \ll 1$ it is observed in [20] that the numerical solution based on the discretization (2.5) seems to be rapidly tending to zero for large $n$ although the corresponding exact solution is not stable. The analysis of this phenomenon leads to the existence of a critical index $n^{*}$ inversely proportional to $1-\lambda$ such that the solution $y_{n}$ of (2.5) displays a tendency to converge to zero before $n=n^{*}$, but it is blowing up after $n=n^{*}$. In other words, numerical calculations have to be accomplished for indices $n$ sufficiently exceeding $n^{*}$ in order to mimic correctly the asymptotic behavior of the exact solution.

Some related essential problems such as the formulation of upper bounds of the solutions of the equation (2.5) as $n \rightarrow \infty$ or discussions on its stability have been the subject matter of the papers [3, 10]. In particular, it follows from the performed analysis that if $|R|+h \eta(\lambda)|S| \leq 1$, where

$$
\eta(\lambda):=\sup _{n \in \mathbb{Z}^{+}}\left(\alpha_{n}+\left|1-\alpha_{n}\right|\right),
$$


then any solution $y_{n}$ of (2.5) is bounded and, moreover, the asymptotic estimate

$$
y_{n}=O\left(n^{-\log _{\lambda} \gamma}\right) \quad \text { as } n \rightarrow \infty, \quad \gamma:=|R|+h \eta(\lambda)|S|
$$

is guaranteed. To make this result effective it is necessary to specify $\eta(\lambda)$. This specification turned out to be a nontrivial task requiring special procedures with respect to rationality or irrationality of $\lambda$. By [3, Theorem 6] the explicit values of $\eta(\lambda), 0<\lambda<1$ are given by the formula

$$
\eta(\lambda)=\left\{\begin{array}{ll}
1, & \lambda=1 / l, \quad l \in\{2,3, \ldots\} \\
1+\lambda-\frac{2}{l}, & \lambda=k / l, \quad k, l \in\{2,3, \ldots\} \\
1+\lambda, & \lambda \text { irrational. }
\end{array}\right. \text { and relatively prime }
$$

Let us emphasize that previous results have been derived in a more general case when the equation (1.1) and its discretization (2.5) involve the neutral term.

The important theoretical question about these numerical approximations is the problem whether the numerical and exact solutions admit a related asymptotic behavior on the unbounded domain. Recall that the qualitative behavior of the solutions of the exact equation (1.1) is well known (see, e.g., [11, 13, 14]). In particular, if Re $a<0$, then the estimate

$$
y(t)=O\left(t^{-\log _{\lambda}|b / a|}\right) \quad \text { as } t \rightarrow \infty
$$

holds for any solution $y$ of (1.1) and, moreover, if $y(t)=o\left(t^{-\log _{\lambda}|b / a|}\right)$ as $t \rightarrow \infty$, then $y$ is the zero solution (in other words, the constant $-\log _{\lambda}|b / a|$ cannot be improved). Comparing the estimates (2.7) and (2.9) of the numerical and exact solutions, respectively, we can find a gap between these upper bounds. Indeed, even if $\eta(\lambda)=1$ (e.g. when $\lambda$ is a reciprocal of an integer), the value $\gamma$ becomes

$$
\gamma=|R|+h|S|=\frac{|2+h a|+2 h|b|}{|2-h a|}
$$

and it requires only a routine calculation to check that $\gamma>|b / a|$ for any $h>0$ provided $-|a|+|b|<0$.

The aim of this paper is, among others, to fill in this gap and present (at least partial) correspondence between the asymptotic behavior of the exact solution and the numerical solution based on the discretization via the trapezoidal rule.

\section{The ASymptotic Behavior of the trapezoidal RUle}

This section presents the main result formulating the upper bound of the solutions $y_{n}$ of (2.1). To describe this asymptotic estimate we introduce the inequality

$$
\left|S_{n}\right| h\left(\left|\beta_{n}\right| \varrho_{\lfloor\lambda n\rfloor}+\left|\alpha_{n}\right| \varrho_{\lfloor\lambda n\rfloor+1}\right) \leq\left(1-\left|R_{n}\right|\right) \varrho_{n}, \quad n=0,1, \ldots,
$$

which plays a key role in our investigations. To simplify the analysis we further assume that

$$
\tilde{S}:=\sup _{n \in \mathbb{Z}^{+}}\left(\left|S_{n}\right|\right)<\infty, \tilde{\eta}:=\sup _{n \in \mathbb{Z}^{+}}\left(\left|\beta_{n}\right|+\left|\alpha_{n}\right|\right)<\infty, \tilde{R}:=\sup _{n \in \mathbb{Z}^{+}}\left(\left|R_{n}\right|\right)<1 .
$$

If we set

$$
\tilde{\gamma}:=\frac{h \tilde{\eta} \tilde{S}}{1-\tilde{R}}
$$

then we can present the explicit form of a solution of (3.1). 
Proposition 3.1. Consider the inequality (3.1) and assume that (3.2) holds. Then the sequence

$$
\varrho_{n}= \begin{cases}\left(n-\frac{1}{1-\lambda}\right)^{-\log _{\lambda} \tilde{\gamma}} & \text { for } \tilde{\gamma} \geq 1, \\ \left(n+\frac{1}{1-\lambda}\right)^{-\log _{\lambda} \tilde{\gamma}} & \text { for } 0<\tilde{\gamma}<1\end{cases}
$$

defines the positive solution of (3.1) for all $n \in \mathbb{Z}^{+}, n \geq 1 /(1-\lambda)$.

Proof. First let $\tilde{\gamma} \geq 1$. Then $\left(\varrho_{n}\right)$ is the nondecreasing sequence and we can write

$$
\left|S_{n}\right| h\left(\left|\beta_{n}\right| \varrho_{\lfloor\lambda n\rfloor}+\left|\alpha_{n}\right| \varrho_{\lfloor\lambda n\rfloor+1}\right) \leq \tilde{S} h \tilde{\eta} \varrho_{\lfloor\lambda n\rfloor+1} .
$$

Substituting the corresponding form of $\varrho_{n}$ one gets

$$
\begin{aligned}
& \tilde{S} h \tilde{\eta}\left(\lfloor\lambda n\rfloor+1-\frac{1}{1-\lambda}\right)^{-\log _{\lambda} \tilde{\gamma}} \leq \tilde{S} h \tilde{\eta}\left(\lambda n-\frac{\lambda}{1-\lambda}\right)^{-\log _{\lambda} \tilde{\gamma}} \\
& =\frac{\tilde{S} h \tilde{\eta}}{\tilde{\gamma}}\left(n-\frac{1}{1-\lambda}\right)^{-\log _{\lambda} \tilde{\gamma}}=(1-\tilde{R})\left(n-\frac{1}{1-\lambda}\right)^{-\log _{\lambda} \tilde{\gamma}}
\end{aligned}
$$

by use of (3.3). The case $0<\tilde{\gamma}<1$ can be dealt with quite similarly.

Now we can state the main assertion formulating the asymptotic estimate of all solutions $y_{n}$ of (2.1).

Theorem 3.2. Let $y_{n}$ be a solution of (2.1), where we assume the validity of the hypothesis (3.2) and let $\tilde{\gamma}$ be given by (3.3). Then

$$
y_{n}=O\left(n^{-\log _{\lambda} \tilde{\gamma}}\right) \quad \text { as } n \rightarrow \infty .
$$

Proof. We introduce the substitution $z_{n}=y_{n} / \varrho_{n}$ in (2.1), where $\varrho_{n}$ is given by (3.4). Then

$$
\varrho_{n+1} z_{n+1}=R_{n} \varrho_{n} z_{n}+S_{n} h\left(\varrho_{\lfloor\lambda n\rfloor} z_{\lfloor\lambda n\rfloor} \beta_{n}+\varrho_{\lfloor\lambda n\rfloor+1} z_{\lfloor\lambda n\rfloor+1} \alpha_{n}\right) .
$$

We aim at showing that every solution $z_{n}$ of (3.6) is bounded as $n \rightarrow \infty$. Choose

$$
\sigma_{0}>\max \left(\frac{1+\lambda}{1-\lambda}, \frac{2-\lambda}{(1-\lambda) \lambda}\right), \quad \sigma_{0} \in \mathbb{Z}^{+}
$$

and define points $\sigma_{m+1}:=\left\lfloor\frac{\sigma_{m}-1}{\lambda}\right\rfloor$, where $m=0,1, \ldots$ The condition (3.7) guarantees that $\sigma_{1}>\sigma_{0}$ and $\varrho_{n}>0$ for $n=\left\lfloor\lambda \sigma_{0}\right\rfloor,\left\lfloor\lambda \sigma_{0}\right\rfloor+1, \ldots$ Moreover, it follows that

$$
\lambda^{-m}\left(\sigma_{0}-\frac{1+\lambda}{1-\lambda}\right) \leq \sigma_{m} \leq \lambda^{-1} \sigma_{m-1}, \quad m=1,2, \ldots .
$$

Further, we introduce intervals $I_{0}:=\left[\left\lfloor\lambda \sigma_{0}\right\rfloor, \sigma_{0}\right] \cap \mathbb{Z}^{+}, I_{m+1}:=\left[\sigma_{m}, \sigma_{m+1}\right] \cap \mathbb{Z}^{+}$ and denote

$$
B_{m}:=\sup \left(\left|z_{k}\right|, k \in \bigcup_{j=0}^{m} I_{j}\right), \quad m=0,1,2 \ldots
$$

Let $n^{\star} \in I_{m+1}, n^{\star}>\sigma_{m}$ be arbitrary. Using the inequality $\left\lfloor\lambda\left(n^{\star}-1\right)\right\rfloor+1 \leq \sigma_{m}$ following from the definition of $\sigma_{m+1}$ we wish to express and estimate $z_{n^{\star}}$ in terms of $z_{k}$, where $k \in \bigcup_{j=0}^{m} I_{j}$. On this account it is necessary to distinguish the following three cases: 
(i) Let $R_{n^{\star}-1}=0$. Then

$$
z_{n^{\star}}=\frac{1}{\varrho_{n^{\star}}} S_{n^{\star}-1} h\left(\varrho_{\left\lfloor\lambda\left(n^{\star}-1\right)\right\rfloor} z_{\left\lfloor\lambda\left(n^{\star}-1\right)\right\rfloor} \beta_{n^{\star}-1}+\varrho_{\left\lfloor\lambda\left(n^{\star}-1\right)\right\rfloor+1} z_{\left\lfloor\lambda\left(n^{\star}-1\right)\right\rfloor+1} \alpha_{n^{\star}-1}\right) .
$$

Taking absolute values we get

$$
\left|z_{n^{\star}}\right| \leq B_{m} \frac{1}{\varrho_{n^{\star}}}\left|S_{n^{\star}-1} h\left(\varrho_{\left\lfloor\lambda\left(n^{\star}-1\right)\right\rfloor} \beta_{n^{\star}-1}+\varrho_{\left\lfloor\lambda\left(n^{\star}-1\right)\right\rfloor+1} \alpha_{n^{\star}-1}\right)\right| .
$$

Then using (3.1) we can estimate $\left|z_{n^{*}}\right|$ as

$$
\left|z_{n^{\star}}\right| \leq \frac{\varrho_{n^{\star}}-1}{\varrho_{n^{\star}}} B_{m}
$$

If $\tilde{\gamma} \geq 1$, then $\left(\varrho_{n}\right)$ is a nondecreasing sequence; hence $\left|z_{n^{\star}}\right| \leq B_{m}$. If $0<\tilde{\gamma}<1$, then we can use (3.4), (3.8) and the binomial formula to derive the relation

$$
\left|z_{n^{\star}}\right| \leq B_{m}\left(1+K_{1} \lambda^{m}\right),
$$

where $K_{1}$ is a positive real constant.

(ii) Let $R_{n} \neq 0$ for any $n \in\left[\sigma_{m}, n^{\star}-1\right] \cap \mathbb{Z}^{+}$. Multiplying equation (3.6) by $\prod_{l=\sigma_{m}}^{n} \frac{1}{R_{l}}$ we obtain

$$
\Delta\left(\varrho_{n} z_{n} \prod_{l=\sigma_{m}}^{n-1} \frac{1}{R_{l}}\right)=S_{n} h\left(\varrho_{\lfloor\lambda n\rfloor} z_{\lfloor\lambda n\rfloor} \beta_{n}+\varrho_{\lfloor\lambda n\rfloor+1} z_{\lfloor\lambda n\rfloor+1} \alpha_{n}\right) \prod_{l=\sigma_{m}}^{n} \frac{1}{R_{l}},
$$

where we put $\prod_{l=k}^{k-1} \frac{1}{R_{l}}=1$ for any $k \in \mathbb{Z}^{+}$. Summing this relation from $\sigma_{m}$ to $n^{\star}-1$ we arrive at

$$
\begin{aligned}
\varrho_{n^{\star}} z_{n^{\star}} \prod_{l=\sigma_{m}}^{n^{\star}-1} \frac{1}{R_{l}}-\varrho_{\sigma_{m}} z_{\sigma_{m}}= & h \sum_{p=\sigma_{m}}^{n^{\star}-1} S_{p}\left(\varrho_{\lfloor\lambda p\rfloor} z_{\lfloor\lambda p\rfloor} \beta_{p}\right. \\
& \left.+\varrho_{\lfloor\lambda p\rfloor+1} z_{\lfloor\lambda p\rfloor+1} \alpha_{p}\right) \prod_{l=\sigma_{m}}^{p} \frac{1}{R_{l}}
\end{aligned}
$$

i.e.

$$
\begin{aligned}
z_{n^{\star}}= & \frac{\varrho_{\sigma_{m}}}{\varrho_{n^{\star}}} z_{\sigma_{m}} \prod_{l=\sigma_{m}}^{n^{\star}-1} R_{l}+\frac{h}{\varrho_{n^{\star}}} \sum_{p=\sigma_{m}}^{n^{\star}-1} S_{p}\left(\varrho_{\lfloor\lambda p\rfloor} z_{\lfloor\lambda p\rfloor} \beta_{p}\right. \\
& \left.+\varrho_{\lfloor\lambda p\rfloor+1} z_{\lfloor\lambda p\rfloor+1} \alpha_{p}\right) \prod_{l=p+1}^{n^{\star}-1} R_{l} .
\end{aligned}
$$

Then

$$
\left|z_{n^{\star}}\right| \leq B_{m}\left(\frac{\varrho_{\sigma_{m}}}{\varrho_{n^{\star}}} \prod_{l=\sigma_{m}}^{n^{\star}-1}\left|R_{l}\right|+\frac{1}{\varrho_{n^{\star}}} \sum_{p=\sigma_{m}}^{n^{\star}-1}\left(1-\left|R_{p}\right|\right) \varrho_{p} \prod_{l=p+1}^{n^{\star}-1}\left|R_{l}\right|\right)
$$

by use of (3.1). Now we consider the obvious identity

$$
\left(1-\left|R_{p}\right|\right) \prod_{l=p+1}^{n^{\star}-1}\left|R_{l}\right|=\Delta \prod_{l=p}^{n^{\star}-1}\left|R_{l}\right| .
$$


Substituting this into (3.11) and summing by parts one gets

$$
\begin{aligned}
\left|z_{n^{\star}}\right| & \leq B_{m}\left(\frac{\varrho_{\sigma_{m}}}{\varrho_{n^{\star}}} \prod_{l=\sigma_{m}}^{n^{\star}-1}\left|R_{l}\right|+\frac{1}{\varrho_{n^{\star}}} \sum_{p=\sigma_{m}}^{n^{\star}-1} \varrho_{p} \Delta \prod_{l=p}^{n^{\star}-1}\left|R_{l}\right|\right) \\
& =B_{m}\left(\frac{\varrho_{\sigma_{m}}}{\varrho_{n^{\star}}} \prod_{l=\sigma_{m}}^{n^{\star}-1}\left|R_{l}\right|+1-\prod_{l=\sigma_{m}}^{n^{\star}-1}\left|R_{l}\right| \frac{\varrho_{\sigma_{m}}}{\varrho_{n^{\star}}}-\sum_{p=\sigma_{m}}^{n^{\star}-1} \prod_{l=p+1}^{n^{\star}-1}\left|R_{l}\right| \frac{\Delta \varrho_{p}}{\varrho_{n^{\star}}}\right) \\
& =B_{m}\left(1-\frac{1}{\varrho_{n^{\star}}} \sum_{p=\sigma_{m}}^{n^{\star}-1} \Delta \varrho_{p} \prod_{l=p+1}^{n^{\star}-1}\left|R_{l}\right|\right) \\
& =B_{m}\left(1-\frac{1}{\varrho_{n^{\star}}} \sum_{p=\sigma_{m}}^{n^{\star}-1} \frac{\Delta \varrho_{p}}{1-\left|R_{p}\right|} \Delta \prod_{l=p}^{n^{\star}-1}\left|R_{l}\right|\right) .
\end{aligned}
$$

If $\tilde{\gamma} \geq 1$, then $\varrho_{p}$ is nondecreasing; hence $\Delta \varrho_{p} \geq 0$ and $\left|z_{n^{\star}}\right| \leq B_{m}$. If $0<\tilde{\gamma}<1$, then $\Delta \varrho_{p}$ is negative and nondecreasing; hence

$$
\begin{aligned}
\left|z_{n^{\star}}\right| & \leq B_{m}\left(1-\frac{\Delta \varrho_{\sigma_{m}}}{\varrho_{n^{\star}}(1-\tilde{R})} \sum_{p=\sigma_{m}}^{n^{\star}-1} \Delta \prod_{l=p}^{n^{\star}-1}\left|R_{l}\right|\right) \\
& =B_{m}\left(1-\frac{\Delta \varrho_{\sigma_{m}}}{\varrho_{n^{\star}}(1-\tilde{R})}\left(1-\prod_{l=\sigma_{m}}^{n^{\star}-1}\left|R_{l}\right|\right)\right) \\
& \leq B_{m}\left(1-\frac{\Delta \varrho_{\sigma_{m}}}{\varrho_{\sigma_{m+1}}(1-\tilde{R})}\right) .
\end{aligned}
$$

Substituting the corresponding form of $\varrho_{n}$ we can derive

$$
\frac{-\Delta \varrho_{\sigma_{m}}}{\varrho_{\sigma_{m+1}}(1-\tilde{R})}=\frac{\left(\sigma_{m}+\frac{1}{1-\lambda}\right)^{-\log _{\lambda} \tilde{\gamma}}\left(1-\left(1+\frac{1}{\sigma_{m}+\frac{1}{1-\lambda}}\right)^{-\log _{\lambda} \tilde{\gamma}}\right)}{(1-\tilde{R})\left(\sigma_{m+1}+\frac{1}{1-\lambda}\right)^{-\log _{\lambda} \tilde{\gamma}}} .
$$

Considering (3.8) and using the binomial formula we arrive at

$$
\frac{-\Delta \varrho_{\sigma_{m}}}{\varrho_{\sigma_{m+1}}(1-\tilde{R})} \leq \frac{\log _{\lambda} \tilde{\gamma}}{\tilde{\gamma}(1-\tilde{R}) \sigma_{m}} \leq K_{2} \lambda^{m}
$$

where

$$
K_{2}=\frac{\log _{\lambda} \tilde{\gamma}}{\tilde{\gamma}(1-\tilde{R})\left(\sigma_{0}-\frac{1+\lambda}{1-\lambda}\right)}>0
$$

Consequently,

$$
\left|z_{n^{\star}}\right| \leq B_{m}\left(1+K_{2} \lambda^{m}\right)
$$

(iii) Let $R_{n^{\star}-1} \neq 0$ and $R_{k}=0$ for some $k \in\left[\sigma_{m}, n^{\star}-2\right] \cap \mathbb{Z}^{+}$. The proof technique applied in this case is a combination of the procedures utilized in cases (i)-(ii), and therefore we present only the main idea. 
First we denote $\sigma^{\star}:=\sup \left(k, k \in\left[\sigma_{m}, n^{\star}-2\right] \cap \mathbb{Z}^{+}\right.$and $\left.R_{k}=0\right)$. Then we multiply equation (3.6) by $\prod_{l=\sigma^{\star}+1}^{n} \frac{1}{R_{l}}$ and sum from $\sigma^{\star}+1$ to $n^{\star}-1$ to obtain

$$
\begin{aligned}
z_{n^{\star}}= & \frac{\varrho_{\sigma^{\star}+1}}{\varrho_{n^{\star}}} z_{\sigma^{\star}+1} \prod_{l=\sigma^{\star}+1}^{n^{\star}-1} R_{l}+\frac{h}{\varrho_{n^{\star}}} \sum_{p=\sigma^{\star}+1}^{n^{\star}-1} S_{p}\left(\varrho_{\lfloor\lambda p\rfloor} z_{\lfloor\lambda p\rfloor} \beta_{p}\right. \\
& \left.+\varrho_{\lfloor\lambda p\rfloor+1} z_{\lfloor\lambda p\rfloor+1} \alpha_{p}\right) \prod_{l=p+1}^{n^{\star}-1} R_{l} .
\end{aligned}
$$

The definition of $\sigma^{\star}$ implies $R_{\sigma^{\star}}=0$; hence by case (i) we can use the estimate

$$
\left|z_{\sigma^{\star}+1}\right| \leq B_{m}\left(1+K_{1} \lambda^{m}\right) .
$$

Then the application of (3.1) yields

$$
\left|z_{n^{\star}}\right| \leq B_{m}\left(1+K_{1} \lambda^{m}\right)\left(\frac{\varrho_{\sigma^{\star}+1}}{\varrho_{n^{\star}}} \prod_{l=\sigma^{\star}+1}^{n^{\star}-1}\left|R_{l}\right|+\frac{1}{\varrho_{n^{\star}}} \sum_{p=\sigma^{\star}+1}^{n^{\star}-1}\left(1-\left|R_{p}\right|\right) \varrho_{p} \prod_{l=p+1}^{n^{\star}-1}\left|R_{l}\right|\right) .
$$

The right-hand side of this inequality is a modification of the corresponding term involved in (3.11) with $\sigma_{m}$ replaced by $\sigma^{*}+1$. Using the same line of arguments as given in case (ii) we arrive at

$$
\left|z_{n^{\star}}\right| \leq B_{m}\left(1+K_{1} \lambda^{m}\right)\left(1+K_{2} \lambda^{m}\right) \leq B_{m}\left(1+K_{3} \lambda^{m}\right),
$$

where $K_{3}$ is a positive real constant.

Summarizing cases (i)-(iii), the estimates (3.10), (3.13) and (3.14) imply that

$$
\left|z_{n^{\star}}\right| \leq B_{m}\left(1+K \lambda^{m}\right) \quad \text { as } m \rightarrow \infty
$$

for arbitrary $n^{\star} \in I_{m+1}, n^{*}>\sigma_{m}$ and a suitable $K>0$. Consequently,

$$
B_{m+1} \leq B_{m}\left(1+K \lambda^{m}\right) \leq B_{0} \prod_{j=0}^{m}\left(1+K \lambda^{j}\right) \leq B_{0} \exp \left\{\frac{K}{1-\lambda}\right\}
$$

and the sequence $\left(B_{m}\right)$ is uniformly bounded. The estimate (3.5) is proved.

Remark 3.3. The significance of the hypothesis (3.2) consists in the fact that it provides an explicit form of a solution $\varrho_{n}$ of the inequality (3.1) and thus enables us to formulate the effective asymptotic criterion for the trapezoidal rule (2.1). Let us emphasize that Theorem 3.2 can be extended to particular cases of (2.1) not satisfying some of the assumptions involved in (3.2).

To outline this possible extension we first assume that $\left|S_{n}\right|$ is a nondecreasing and unbounded sequence, i.e. $\tilde{S}=\infty$ (the validity of other assumptions of (3.2) remains preserved). Then the inequality (3.1) always admits a positive and increasing solution $\varrho_{n}$. Indeed, e.g., the sequence

$$
\varrho_{n}=\left(\eta\left|S_{n}\right| /(1-\tilde{R})\right)^{n}
$$

satisfies (3.1) for all $n$ large enough. Now it is easy to verify that the technique applied in the proof of Theorem 3.2 is utilizable also provided such a solution $\varrho_{n}$ is considered instead of (3.4). In particular, $\Delta \varrho_{n}>0$ for all $n$ large enough and we can omit the parts of the proof discussing the asymptotically stable case. Then the asymptotic bound (3.5) presented in Theorem 3.2 can be slightly modified as

$$
y_{n}=O\left(\varrho_{n}\right) \quad \text { as } n \rightarrow \infty .
$$


Of course, this asymptotic estimate (with the above specified $\varrho_{n}$ ) may be too rough in particular cases. Then, considering a concrete equation, we can try to find a more suitable (positive and increasing) solution $\varrho_{n}$ of (3.1) representing the stronger upper bound sequence for the estimate (3.16). The illustration of this procedure is given in Example 3.4.

Similarly we can discuss the case $\tilde{\eta}=\infty$ as well as the case $\tilde{S}=\tilde{\eta}=\infty$. The possible omission of the last condition of (3.2), namely $\tilde{R}<1$, is the most interesting point. First note that if $\left|R_{n}\right| \geq 1$ for all $n$ sufficiently large, then the inequality (3.1) does not admit any positive solution $\varrho_{n}$. In particular, if $a_{n} \equiv a$ is a constant, then the assumption $\tilde{R}<1$ (which is satisfied if and only if Re $a<0$ ) cannot be omitted. If $a_{n}$ is not a constant, then we can consider the case where $\left|R_{n}\right|<1$ for all $n$ sufficiently large and $\lim _{n \rightarrow \infty}\left|R_{n}\right|=1$, i.e. $\tilde{R}=1$. Under some particular choices of $b_{n}$ the inequality (3.1) can admit a positive and nondecreasing solution $\varrho_{n}$; hence the estimate (3.16) remains valid. In particular, if we substitute $\varrho_{n} \equiv$ const into (3.1), then we obtain the inequality

$$
\left|S_{n}\right| h\left(\left|\beta_{n}\right|+\left|\alpha_{n}\right|\right) \leq 1-\left|R_{n}\right|, \quad n=0,1, \ldots,
$$

which is the condition guaranteeing (without assuming (3.2) ) the stability of the discretization (2.1). The case where $\varrho_{n}$ decreases is much more complicated. Besides the determination of the form of $\varrho_{n}$ we have to verify some additional nontrivial requirements on $\varrho_{n}$ and $a_{n}$ following from calculations performed in the corresponding part of the proof of Theorem 3.2 .

To summarize, in particular cases the omission of some assumptions involved in the hypothesis (3.2) is possible, but searching for a suitable solution $\varrho_{n}$ of (3.1) without assuming (3.2) is, in general, a difficult task (especially in the asymptotically stable case).

The following example illustrates the extension of Theorem 3.2 to the case where the assumption $\tilde{S}<\infty$ is not satisfied.

Example 3.4. We consider the differential equation

$$
y^{\prime}(t)=a y(t)+b t y(t / 2), \quad t \geq 0,
$$

where $a<0$ and $b \neq 0$ are real scalars. The discretization of this equation based on the trapezoidal rule (2.1) yields the recurrence relation

$$
y_{n+1}=R y_{n}+S_{n} h\left(\beta_{n} y_{\lfloor n / 2\rfloor}+\alpha_{n} y_{\lfloor n / 2\rfloor+1}\right)
$$

with

and

$$
R=\frac{2+h a}{2-h a}, \quad S_{n}=\frac{2 b n h}{2-h a}
$$

$$
\alpha_{n}=\left\{\begin{array}{ll}
\frac{1}{4}+\frac{1}{4 n}, & n \text { is even, } \\
\frac{3}{4}+\frac{1}{2 n}, & n \text { is odd, }
\end{array} \quad \beta_{n}= \begin{cases}\frac{3}{4}+\frac{1}{4 n}, & n \text { is even, } \\
\frac{1}{4}, & n \text { is odd },\end{cases}\right.
$$

i.e. $\alpha_{n}+\beta_{n}=1+\frac{1}{2 n}$ for all $n=1,2, \ldots$. Although the assumption $\tilde{S}<\infty$ involved in the hypothesis (3.2) is not satisfied, we outline the applicability of Theorem 3.2 regardless of the invalidity of (3.2). It is enough to find an appropriate solution of the inequality

$$
\left|S_{n}\right| h\left(\beta_{n} \varrho_{\lfloor n / 2\rfloor}+\alpha_{n} \varrho_{\lfloor n / 2\rfloor+1}\right) \leq(1-|R|) \varrho_{n}
$$


resulting from (3.1). On this account we consider the auxiliary functional equation

$$
p t \varphi\left(\frac{t}{2}\right)=q \varphi(t), \quad t>0
$$

where $p, q>0$ are real scalars, which turns out to be of key importance in this investigation. To our knowledge, one of the first papers discussing this equation was that of [6]. Utilizing the Mellin transform method, the searched solution $\varphi$ was derived in the form

$$
\varphi(t)=t^{\log _{2} \frac{p}{q}+\frac{1}{2}\left(\log _{2} t+1\right)} .
$$

This relation (with $p=2|b| h^{2} /|2-h a|$ and $q=1-|2+h a| /|2-h a|$ ) can be only slightly modified to obtain the form

$$
\varrho_{n}=\frac{n-\frac{9}{2}}{n-2}(n-2)^{\log _{2} \frac{p}{q}+\frac{1}{2}\left(\log _{2}(n-2)+1\right)}
$$

defining the required solution of (3.18) for $n \geq 5$. Indeed, since $\varrho_{n}$ is eventually increasing and $\alpha_{n}+\beta_{n}=1+\frac{1}{2 n}$ we can simplify the inequality (3.18) as

$$
\left|S_{n}\right| h\left(1+\frac{1}{2 n}\right) \varrho_{\lfloor n / 2\rfloor+1} \leq(1-|R|) \varrho_{n} .
$$

Then substituting (3.19) into this relation and using some straightforward calculations one can check the validity of this inequality.

Then Theorem 3.2 with respect to Remark 3.3 implies that

$$
y_{n}=O\left(n^{\log _{2} \frac{2|b| h^{2}}{|2-h a|-|2+h a|}+\frac{1}{2}\left(\log _{2} n+1\right)}\right) \quad \text { as } n \rightarrow \infty
$$

for any solution $y_{n}$ of (3.17).

Our next aim is to reformulate Theorem 3.2 in the most important particular case, namely when $a_{n} \equiv a$ and $b_{n} \equiv b$ are complex constants. Hence we take into our considerations the autonomous pantograph equation (1.1) and its discretization (2.5). Applying Theorem 3.2 it is easy to specify the meaning of the symbols $\tilde{S}, \tilde{\eta}$ and $\tilde{R}$ occurring in the hypothesis (3.2). Obviously $\tilde{S}=|S|, \tilde{R}=|R|$ and $\tilde{\eta}=\eta(\lambda)$, where the explicit values of $\eta(\lambda)$ are given by (2.8). Moreover, the condition $|R|<1$ holds if and only if Re $a<0$. We note that this inequality together with the relation $|a|>|b|$ represents the asymptotic stability condition for the exact equation (1.1) (see [11, 14]).

Since the remaining assumptions stated in (3.2) become trivial, we arrive at the following

Corollary 3.5. Let $y_{n}$ be a solution sequence of the discretization (2.5)-(2.6), where Re $a<0, b \neq 0$ and $0<\lambda<1$. Then

$$
y_{n}=O\left(n^{-\log _{\lambda} \tilde{\gamma}}\right) \quad \text { as } n \rightarrow \infty, \quad \tilde{\gamma}=\frac{h \eta(\lambda)|S|}{1-|R|} .
$$

Remark 3.6. We can easily compare the relation (3.20) with the asymptotic estimate (2.7) derived in [3] under the assumption $\gamma \leq 1$. Considering this inequality we get

$$
\tilde{\gamma}=\frac{h \eta(\lambda)|S|}{1-|R|} \leq|R|+h \eta(\lambda)|S|=\gamma
$$


where the equality sign between $\tilde{\gamma}$ and $\gamma$ occurs if and only if $\gamma=1$. In other words, substituting the values $R$ and $S$ from (2.6) into the inequality $\tilde{\gamma} \leq 1$ we can easily check that the solution sequence of (2.5) is bounded if

$$
\text { Re } a<0, \quad \eta(\lambda)|b|+\frac{4 \operatorname{Re} a}{|2+h a|+|2-h a|} \leq 0,
$$

which is the same stability condition as the one derived in [3] and [10] by use of the inequality $\gamma \leq 1$. However, considering the asymptotically stable case $(\gamma<1)$, the formula (3.20) provides a stronger asymptotic estimate than the formula (2.7) yields. More precisely, both formulae affirm the algebraic decay of $y_{n}$, but the asymptotic property (3.20) guarantees the stronger decay rate.

Then the natural question arises, namely what is the relation between the upper bound (2.9) derived in [11, 13, 14, for the exact solution of (1.1) and our upper bound (3.20) derived for its numerical solution. The discussions of this type are very frequent in numerical investigations of the pantograph equation and can be found in many of the above cited papers. In particular, closely related to our question is the paper [19. discussing (among others) qualitative properties of the $\theta$-method for the pantograph equation. As is observed in this paper, we need to choose an appropriate numerical method so that the numerical solution can retain as many of these (asymptotic) properties (of the exact solution) as possible. Note that one of these asymptotic properties discussed in [19] is just the asymptotic formula (2.9); assuming $\operatorname{Re} a<0$ and $|a|>|b|$ it is reported that the numerical solution of (1.1) by the backward Euler method has asymptotically the same decay rate as the exact solution.

Answering our question we first consider the case where $a$ is a real constant ( $b$ can be complex). Then we can observe that $\tilde{\gamma}$ occurring in (3.20) becomes

$$
\tilde{\gamma}= \begin{cases}|b / a| \eta(\lambda) & \text { for } h|a| \leq 2 \\ h|b| \eta(\lambda) / 2 & \text { for } h|a|>2\end{cases}
$$

Hence the value $|b / a|$ known from the asymptotic description of the exact scalar pantograph becomes $|b / a| \eta(\lambda)$ under the discretization (2.5) with the modest restriction on the stepsize $h$. In particular, if $\lambda$ is a reciprocal of an integer, then both the exact solution and the numerical solution by the trapezoidal rule have exactly the same decay rate.

Now we consider the case where both parameters $a, b$ are complex. If Im $a \neq 0$, then the previous relation for $\tilde{\gamma}$ is no longer valid and it turns out that $\tilde{\gamma}$ is always greater than $|b / a| \eta(\lambda)$ and $h|b| \eta(\lambda) / 2$ provided $h|a| \leq 2$ and $h|a|>2$, respectively.

Example 3.7. We consider the initial value problem

$$
y^{\prime}(t)=a y(t)+b y(t / 2), \quad y(0)=1,
$$

where $a<0$ and $b \neq 0$ are real scalars. The trapezoidal rule (2.5) applied to (3.21) becomes

$$
\begin{aligned}
y_{n+1} & = \begin{cases}R y_{n}+\frac{1}{4} h S\left(y_{\frac{n-1}{2}}+3 y_{\frac{n+1}{2}}\right), & n \text { is positive and odd, } \\
R y_{n}+\frac{1}{4} h S\left(3 y_{\frac{n}{2}}+y_{\frac{n}{2}+1}\right), & n \text { is nonnegative and even, }\end{cases} \\
y_{0} & =1,
\end{aligned}
$$

where the symbols $R$ and $S$ have been introduced in (2.6). The formula (3.22) has been thoroughly discussed in [10] especially with respect to related stability properties of (3.22) and the corresponding exact equation (3.21). Applying Corollary 3.5 
we can extend the performed analysis of (3.22) and formulate the upper bound

$$
y_{n}=O\left(n^{\log _{2}|b / a|}\right) \quad \text { as } n \rightarrow \infty,
$$

which is valid for the solution $y_{n}$ of (3.22) provided $2+h a \geq 0$. We emphasize that this estimate coincides with the asymptotics of the exact pantograph equation (3.21) described, e.g., in [13.

In the next two examples we specify the parameters $a, b$ in (3.21) and discuss the upper bound for the trapezoidal rule (3.22) with the stepsize $h=0.05$.

Example 3.8. First we choose $a=-1$ and $b=-0.5$ in (3.21); i.e., we consider the initial value problem

$$
y^{\prime}(t)=-y(t)-0.5 y(t / 2), \quad y(0)=1, \quad t \geq 0 .
$$

Then

$$
\gamma=|R|+h \eta(\lambda)|S| \approx 0.9756, \quad \tilde{\gamma}=\frac{h \eta(\lambda)|S|}{1-|R|}=\left|\frac{b}{a}\right|=0.5
$$

and the asymptotic estimates (2.7) and (3.20) become

$$
y_{n}=O\left(n^{-0.0356}\right) \quad \text { as } n \rightarrow \infty
$$

and

$$
y_{n}=O\left(n^{-1}\right) \quad \text { as } n \rightarrow \infty,
$$

respectively. We emphasize that (3.25) presents the same decay rate for the numerical solution as the corresponding exact solution admits. Our next intention is the computational presentation of the estimate (3.25) and its graphic comparisons with the estimate (3.24) as well as with the real behavior of the discretization (3.22). To make the estimate (3.25) more applicable from the computational viewpoint it is necessary to follow some steps performed in the proof of Theorem 3.2 and specify the $O$-term in (3.25), i.e. determine a constant $L_{1}>0$ such that

$$
\left|y_{n}\right| \leq L_{1} n^{-1} \quad \text { for all } n \text { large enough. }
$$

It follows from the proof of Theorem 3.2 (part (ii) and the relation (3.15) with respect to $K=K_{2}$ ) that $L_{1}=B_{0} \exp \left\{\frac{K_{2}}{1-\lambda}\right\}$, where the constants $B_{0}$ and $K_{2}$ can be calculated via (3.9) and (3.12) as

$$
B_{0}=\sup \left(\left|y_{n}(n+2)\right|, n \in\left[\left\lfloor\frac{\sigma_{0}}{2}\right\rfloor, \sigma_{0}\right] \cap \mathbb{Z}^{+}\right), \quad K_{2}=\frac{41}{\sigma_{0}-3} .
$$

Now we choose $\sigma_{0}$ representing the starting point for the asymptotic estimation performed in the proof of Theorem 3.2. By (3.7), it is enough to put $\sigma_{0}=7$. However, to obtain a reasonable computational and especially graphic illustration of (3.25), we suggest the choice of a larger value of $\sigma_{0}$, say $\sigma_{0}=150$. Then $K_{2} \approx 0.279$ and for the specification of $B_{0}$ it remains to determine (or at least estimate) the values of $y_{n}$ for $n=1,2, \ldots, 150$. By [19, Theorem 2], these values are uniformly bounded by $|y(0)|=1$. However, to obtain a stronger majorant constant $L_{1}$, we prefer their direct calculation via (3.22). Then $B_{0} \approx 1.9369$; hence $L_{1} \approx 3.3834$ and we can make precise the upper bound (3.25) for the solution sequence $y_{n}$ of (3.22) in the form

$$
\left|y_{n}\right| \leq 3.3834 n^{-1} \quad \text { for all } n \text { large enough }
$$

(more precisely, for $n=150,151, \ldots$ ). 
Now we consider the estimate (3.24). Since $y_{\sigma_{0}}=y_{150} \approx 0.012$, we can choose the corresponding majorant constant $L_{2}$ specifying the $O$-term in (3.24) as $L_{2}=$ $0.012 \times 150^{0.0356} \approx 0.0144$ (in other words, to obtain a sharp majorant constant we choose such an $L_{2}$ that the values of $y_{n}$ and its estimate $L_{2} n^{-0.0356}$ coincide for $n=150)$. This implies

$$
\left|y_{n}\right| \leq 0.0144 n^{-0.0356} \quad \text { for all } n \text { large enough }
$$

(in the sequel we can see that this estimate holds for $n=150,151, \ldots$ ).

Now the gap between both asymptotic results can be simply illustrated by Figure 1 . We denote here by $y^{h}(t)$ the linear interpolation of $\left(y_{n}\right)_{n=0}^{n=\infty}$, i.e.

$$
y^{h}(t)=\frac{(n+1) h-t}{h} y_{n}+\frac{t-n h}{h} y_{n+1}, \quad t \in[n h,(n+1) h], \quad n=0,1,2, \ldots,
$$

and consider $t \in[7.5,400]$ (note that the left endpoint $t=7.5$ corresponds to the starting index $\sigma_{0}=150$ of the asymptotic estimation via the relation $t=150 \mathrm{~h}$ ). Figure 1 plots the numerical solution $y^{h}$ of (3.23) as well as its upper bounds $g(t)=3.3834 h t^{-1} \approx 0.1692 t^{-1}$ and $f(t)=0.0144 h^{0.0356} t^{-0.0356} \approx 0.0129 t^{-0.0356}$.

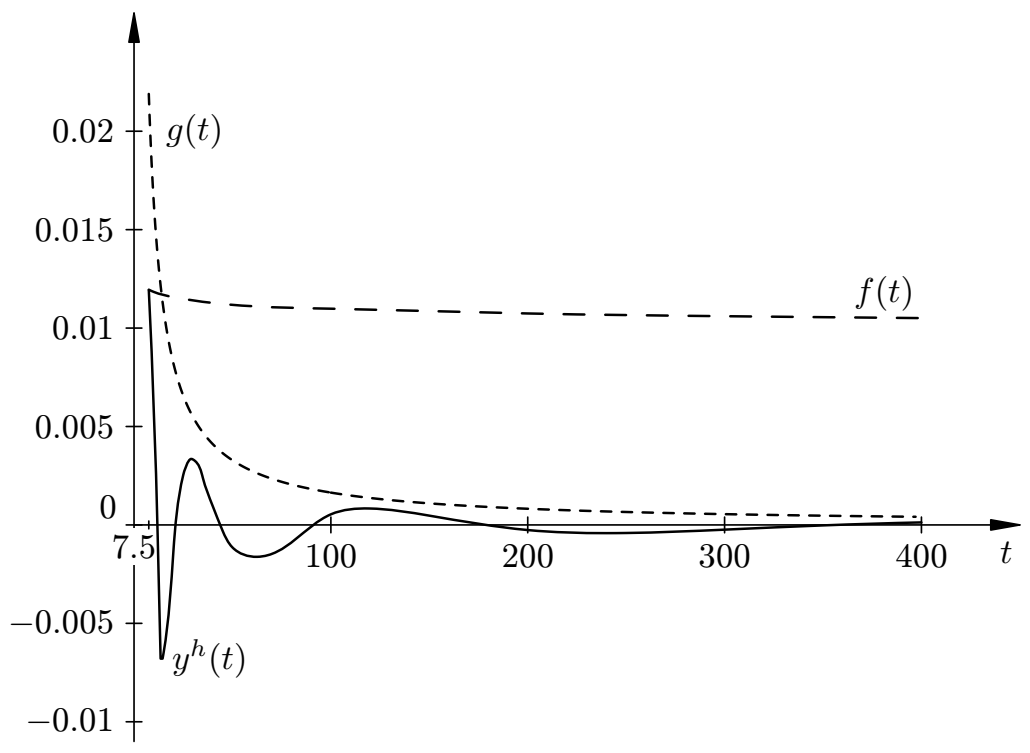

Figure 1. The solution $y^{h}$ and its upper bounds

Example 3.9. Now we illustrate the unstable case of equation (3.21) by the choice $a=-0.5$ and $b=-2$. Consequently, we investigate the initial value problem

$$
y^{\prime}(t)=-0.5 y(t)-2 y(t / 2), \quad y(0)=1, \quad t \geq 0 .
$$

Then $\tilde{\gamma}=\frac{h \eta(\lambda)|S|}{1-|R|}=\left|\frac{b}{a}\right|=4$ and we can rewrite (3.20) as

$$
\left|y_{n}\right| \leq L_{3} n^{2} \quad \text { for all } n \text { large enough, }
$$

where $L_{3}>0$ is a suitable real constant. Following the same procedure as in the previous case and noticing that $\tilde{\gamma}>1$ it follows that $L_{3}=B_{0}$, where

$$
B_{0}=\sup \left(\left|y_{n}(n-2)^{-2}\right|, n \in\left[\left\lfloor\frac{\sigma_{0}}{2}\right\rfloor, \sigma_{0}\right] \cap \mathbb{Z}^{+}\right) .
$$


To obtain a satisfactory graphic illustration of our estimate we can choose, e.g., the same value of $\sigma_{0}$ as in Example 3.8, i.e. $\sigma_{0}=150$. Then $B_{0} \approx 0.000055$, and the asymptotic estimate (3.27) becomes

$$
\left|y_{n}\right| \leq 0.000055 n^{2} \quad \text { for all } n \text { large enough . }
$$

For the sake of completeness we note that, considering the unstable case, we cannot employ the upper bound (2.7) because its validity is restricted by the stability condition $\gamma \leq 1$ (see [3]).

Now we consider the numerical solution $y^{h}(t)$ of the equation (3.26), where $t \in[7.5,400]$ and its upper bound $g(t)=0.000055 t^{2} / h^{2} \approx 0.022007 t^{2}$. Figure 2 plots $\left(t, \log _{10}\left(\left|y^{h}(t)\right|+\epsilon\right)\right)$ and $\left(t, \log _{10} g(t)\right)$, where $\varepsilon=2.23 \times 10^{-308}$.

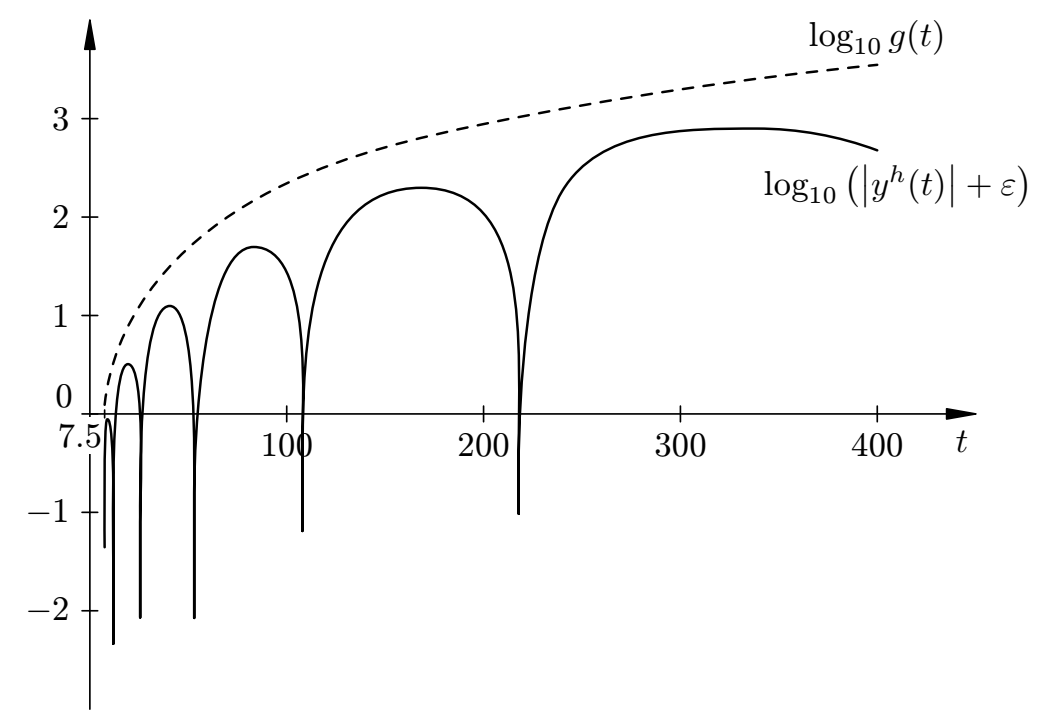

FiguRE 2

\section{Some GEneralizations}

In this section we discuss possible extensions of Theorem 3.2 and a related proof technique. Some of these extensions are quite straightforward (e.g., the involvement of several proportional delays into our considerations), while others require some additional operations. We focus especially on the asymptotic investigation of the trapezoidal rule

$$
y_{n+1}=R_{n} y_{n}+S_{n} h\left(\beta_{n} y_{\left\lfloor\bar{\theta}_{n}\right\rfloor}+\alpha_{n} y_{\left\lfloor\bar{\theta}_{n}\right\rfloor+1}\right),
$$

which originates from the discretization of the differential equation

$$
y^{\prime}(t)=a(t) y(t)+b(t) y(\theta(t)), \quad t \geq t_{0},
$$

involving a general delayed argument (see Section 2). 
To analyse the asymptotics of (4.1), we have to appropriately modify the key inequality (3.1). As might be expected, the relation

$$
\left|S_{n}\right| h\left(\left|\beta_{n}\right| \varrho_{\left\lfloor\bar{\theta}_{n}\right\rfloor}+\left|\alpha_{n}\right| \varrho_{\left\lfloor\bar{\theta}_{n}\right\rfloor+1}\right) \leq\left(1-\left|R_{n}\right|\right) \varrho_{n}, \quad n=0,1, \ldots
$$

seems to be the natural replacement of (3.1). To confirm this conjecture we start by searching for a suitable solution of (4.3). On this account we consider the auxiliary functional equation

$$
\varphi(\theta(t))=\kappa \varphi(t), \quad \kappa=\theta^{\prime}\left(t_{0}\right), \quad t \geq t_{0},
$$

which is usually referred to as the Schröder equation. It is known (see, e.g., [16]) that if $\theta \in C^{2}\left(\left[t_{0}, \infty\right)\right), \theta\left(t_{0}\right)=t_{0}, \theta(t)<t$ for all $t>t_{0}, \theta^{\prime}$ is positive on $\left[t_{0}, \infty\right)$ and $\theta^{\prime}\left(t_{0}\right)<1$, then there exists a unique strictly increasing and continuously differentiable solution $\varphi$ of (4.4) satisfying $\varphi^{\prime}\left(t_{0}\right)=1$. This solution is given by the formula

$$
\varphi(t)=\lim _{n \rightarrow \infty} \kappa^{-n}\left(\theta^{n}(t)-t_{0}\right), \quad t \geq t_{0},
$$

where $\theta^{n}$ means the $n$-th iterate of $\theta$. In the sequel we mention a slightly modified version of this result, where a further condition on $\theta$ (namely $\theta^{\prime}$ nonincreasing) is imposed to ensure some additional properties of $\varphi$. We utilize these properties in the proof of the main result of this section.

Proposition 4.1. Let $\theta \in C^{2}\left(\left[t_{0}, \infty\right)\right)$ be such that $\theta\left(t_{0}\right)=t_{0}, \theta(t)<t$ for all $t>t_{0}, \theta^{\prime}$ is positive and nonincreasing on $\left[t_{0}, \infty\right)$ and $\theta^{\prime}\left(t_{0}\right)<1$. Then the function $\varphi$ defined by (4.5) is the solution of (4.4) such that $\varphi^{\prime}$ is positive, continuous and nonincreasing on $\left[t_{0}, \infty\right)$ and, furthermore, $\varphi^{\prime}(t) / \varphi(t) \leq 1 /\left(t-t_{0}\right)$ for all $t>t_{0}$.

Proof. Differentiating (4.4) one can obtain

$$
\varphi^{\prime}(\theta(t)) \theta^{\prime}(t)=\kappa \varphi^{\prime}(t), \quad t \geq t_{0},
$$

which implies that $\varphi^{\prime}$ is positive and nonincreasing. Similarly,

$$
\frac{\varphi^{\prime}(t)}{\varphi(t)}=\frac{\varphi^{\prime}(t)}{\varphi(t)-\varphi\left(t_{0}\right)} \leq \frac{\varphi^{\prime}(t)}{\varphi^{\prime}(t)\left(t-t_{0}\right)}=\frac{1}{t-t_{0}}, \quad t>t_{0} .
$$

Throughout this section we shall assume that all the assumptions imposed on $\theta$ in Proposition 4.1 are satisfied and that $\varphi$ is the function defined by (4.5) with the properties guaranteed by Proposition 4.1. Then we consider the differential equation (4.2), its trapezoidal discretization (4.1) and the inequality (4.3). To formulate the upper bound of the solutions of (4.1), it is necessary to present the exact form of the solutions of (4.3).

Proposition 4.2. Consider the inequality (4.3) and assume that (3.2) holds. Further, let $t^{*} \geq t_{0}$ be a (unique) real root of the equation $t-\theta(t)=h$ and let $k^{*}=\left\lfloor\left(t^{*}-t_{0}\right) / h\right\rfloor+1$. Then

$$
\varrho_{n}= \begin{cases}\left(\varphi\left(t_{0}+\left(n-k^{*}\right) h\right)\right)^{-\log _{\kappa} \tilde{\gamma}} & \text { for } \tilde{\gamma} \geq 1, \\ \left(\varphi\left(t_{0}+\left(n+k^{*}\right) h\right)\right)^{-\log _{\kappa} \tilde{\gamma}} & \text { for } 0<\tilde{\gamma}<1,\end{cases}
$$

where $\tilde{\gamma}$ is given by (3.3), defines the solution of (4.3). Moreover, if $\tilde{\gamma} \geq 1$, then $\Delta \varrho_{n}$ is nonnegative and if $0<\tilde{\gamma}<1$, then $\Delta \varrho_{n}$ is negative and nondecreasing. 
Proof. First let $\tilde{\gamma} \geq 1$. Then $\varrho_{n}$ is nondecreasing and

$$
\begin{aligned}
\left|S_{n}\right| h\left(\left|\beta_{n}\right| \varrho_{\left\lfloor\bar{\theta}_{n}\right\rfloor}+\left|\alpha_{n}\right| \varrho_{\left\lfloor\bar{\theta}_{n}\right\rfloor+1}\right) & \leq \tilde{S} h \tilde{\eta} \varrho_{\left\lfloor\bar{\theta}_{n}\right\rfloor+1} \\
& \leq \tilde{S} h \tilde{\eta}\left(\varphi\left(t_{0}+\bar{\theta}_{n} h+h-k^{*} h\right)\right)^{-\log _{\kappa} \tilde{\gamma}} \\
& =\tilde{S} h \tilde{\eta}\left(\varphi\left(\theta_{n}+h-k^{*} h\right)\right)^{-\log _{\kappa} \tilde{\gamma}} \\
& \leq \tilde{S} h \tilde{\eta}\left(\varphi\left(\theta_{n-k^{*}}\right)\right)^{-\log _{\kappa} \tilde{\gamma}}=(1-\tilde{R}) \varrho_{n}
\end{aligned}
$$

by use of (4.4).

The case $0<\tilde{\gamma}<1$ can be dealt with quite similarly. Moreover, the additional properties of $\Delta \varrho_{n}$ follow from the corresponding properties of $\varphi$.

Remark 4.3. The sequence (4.6) is defined for all $n \geq k^{*}$ provided $\tilde{\gamma} \geq 1$. If $0<\tilde{\gamma}<1$, then $\varrho_{n}$ defines the solution of (4.3) for all $n \geq 0$.

Now we can formulate the following generalization of Theorem 3.2

Theorem 4.4. Let $y_{n}$ be a solution of (4.1), where we assume the validity of the hypothesis (3.2), let $\tilde{\gamma}$ be given by (3.3) and let $\kappa=\theta^{\prime}\left(t_{0}\right)$. Then

$$
y_{n}=O\left((\varphi(n))^{-\log _{\kappa} \tilde{\gamma}}\right) \quad \text { as } n \rightarrow \infty .
$$

Proof. The method of proof is a modification of the procedure utilized in the proof of Theorem 3.2. First we introduce the substitution $z_{n}=y_{n} / \varrho_{n}$, where $\varrho_{n}$ is given by (4.6). Then

$$
\varrho_{n+1} z_{n+1}=R_{n} \varrho_{n} z_{n}+S_{n} h\left(\varrho_{\left\lfloor\bar{\theta}_{n}\right\rfloor} z_{\left\lfloor\bar{\theta}_{n}\right\rfloor} \beta_{n}+\varrho_{\left\lfloor\bar{\theta}_{n}\right\rfloor+1} z_{\left\lfloor\bar{\theta}_{n}\right\rfloor+1} \alpha_{n}\right) .
$$

Choose $\sigma_{0}>\max \left(\frac{1+\kappa}{1-\kappa}, \frac{\theta^{-1}\left(t_{0}+k^{*} h\right)-t_{0}}{h}\right), \sigma_{0} \in \mathbb{Z}^{+}$and define $I_{0}:=\left[\left\lfloor\bar{\theta}_{\sigma_{0}}\right\rfloor, \sigma_{0}\right] \cap \mathbb{Z}^{+}$, $\sigma_{m+1}:=\left\lfloor\frac{\theta^{-1}\left(t_{0}+\left(\sigma_{m}-1\right) h\right)-t_{0}}{h}\right\rfloor, I_{m+1}:=\left[\sigma_{m}, \sigma_{m+1}\right] \cap \mathbb{Z}^{+}, B_{m}:=\sup \left(\left|z_{k}\right|, k \in\right.$ $\left.\bigcup_{j=0}^{m} I_{j}\right), m=0,1, \ldots$. Now considering arbitrary $n^{*} \in I_{m+1}, n^{*}>\sigma_{m}$ we distinguish the following cases:

(i) Let $R_{n^{\star}-1}=0$. Using the same line of arguments as given in the proof of Theorem 3.2 we arrive at the estimate

$$
\left|z_{n^{\star}}\right| \leq \frac{\varrho_{n^{\star}-1}}{\varrho_{n^{\star}}} B_{m}
$$

If $\tilde{\gamma} \geq 1$, then $\left|z_{n^{\star}}\right| \leq B_{m}$. If $0<\tilde{\gamma}<1$, then we utilize the mean value theorem, the binomial formula and properties of $\varphi$ guaranteed by Proposition 4.1 to rewrite the term $\varrho_{n^{*}-1} / \varrho_{n^{*}}$ as

$$
\begin{aligned}
\frac{\varrho_{n^{*}-1}}{\varrho_{n^{*}}} & =\left(\frac{\varphi\left(t_{0}+\left(n^{*}+k^{*}\right) h\right)}{\varphi\left(t_{0}+\left(n^{*}-1+k^{*}\right) h\right)}\right)^{\log _{\kappa} \tilde{\gamma}} \\
& =\left(1+\frac{\varphi\left(t_{0}+\left(n^{*}+k^{*}\right) h\right)-\varphi\left(t_{0}+\left(n^{*}-1+k^{*}\right) h\right)}{\varphi\left(t_{0}+\left(n^{*}-1+k^{*}\right) h\right)}\right)^{\log _{\kappa} \tilde{\gamma}} \\
& \leq\left(1+h \frac{\varphi^{\prime}\left(t_{0}+\left(n^{*}-1+k^{*}\right) h\right)}{\varphi\left(t_{0}+\left(n^{*}-1+k^{*}\right) h\right)}\right)^{\log _{\kappa} \tilde{\gamma}} \leq\left(1+\frac{1}{\sigma_{m}}\right)^{\log _{\kappa} \tilde{\gamma}} \leq 1+\frac{K_{1}}{\sigma_{m}},
\end{aligned}
$$

where $K_{1}$ is a positive real constant. Consequently,

$$
\left|z_{n^{\star}}\right| \leq B_{m}\left(1+\frac{K_{1}}{\sigma_{m}}\right) .
$$


(ii) Let $R_{n} \neq 0$ for any $n \in\left[\sigma_{m}, n^{\star}-1\right] \cap \mathbb{Z}^{+}$. Applying the corresponding steps performed in the proof of Theorem 3.2 we can derive the estimate

$$
\left|z_{n^{*}}\right| \leq B_{m}\left(1-\frac{1}{\varrho_{n^{\star}}} \sum_{p=\sigma_{m}}^{n^{\star}-1} \frac{\Delta \varrho_{p}}{1-\left|R_{p}\right|} \Delta \prod_{l=p}^{n^{\star}-1}\left|R_{l}\right|\right)
$$

If $\tilde{\gamma} \geq 1$, then $\left|z_{n^{\star}}\right| \leq B_{m}$. If $0<\tilde{\gamma}<1$, then, by Proposition 4.2. $\Delta \varrho_{p}$ is negative and nondecreasing; hence

$$
\left|z_{n^{\star}}\right| \leq B_{m}\left(1-\frac{\Delta \varrho_{\sigma_{m}}}{\varrho_{\sigma_{m+1}}(1-\tilde{R})}\right)
$$

To estimate the ratio term we use the mean value theorem and the monotonicity of $\varphi^{\prime}$ to obtain

$$
\begin{aligned}
-\Delta \varrho_{\sigma_{m}} & =\left(\varphi\left(t_{0}+\left(\sigma_{m}+k^{*}\right) h\right)\right)^{-\log _{\kappa} \tilde{\gamma}}-\left(\varphi\left(t_{0}+\left(\sigma_{m}+1+k^{*}\right) h\right)\right)^{-\log _{\kappa} \tilde{\gamma}} \\
& \leq h \log _{\kappa} \tilde{\gamma}\left(\varphi\left(t_{0}+\left(\sigma_{m}+k^{*}\right) h\right)\right)^{-\log _{\kappa} \tilde{\gamma}-1} \varphi^{\prime}\left(t_{0}+\left(\sigma_{m}+k^{*}\right) h\right) .
\end{aligned}
$$

Similarly,

$$
\begin{aligned}
\varrho_{\sigma_{m+1}} & =\left(\varphi\left(t_{0}+\left(\sigma_{m+1}+k^{*}\right) h\right)\right)^{-\log _{\kappa} \tilde{\gamma}} \\
& \geq\left(\varphi\left(\theta^{-1}\left(t_{0}+\left(\sigma_{m}-1\right) h\right)+k^{*} h\right)\right)^{-\log _{\kappa} \tilde{\gamma}} \\
& \geq\left(C \varphi\left(\theta^{-1}\left(t_{0}+\left(\sigma_{m}-1\right) h\right)\right)\right)^{-\log _{\kappa} \tilde{\gamma}} \\
& \geq\left(\frac{C}{\kappa}\right)^{-\log _{\kappa} \tilde{\gamma}}\left(\varphi\left(t_{0}+\left(\sigma_{m}+k^{*}\right) h\right)\right)^{-\log _{\kappa} \tilde{\gamma}}
\end{aligned}
$$

by use of (4.4), $C$ being a suitable positive real constant. Consequently,

$$
\frac{-\Delta \varrho_{\sigma_{m}}}{\varrho_{\sigma_{m+1}}(1-\tilde{R})} \leq\left(\frac{C}{\kappa}\right)^{\log _{\kappa} \tilde{\gamma}} \frac{h \log _{\kappa} \tilde{\gamma}}{1-\tilde{R}} \frac{\varphi^{\prime}\left(t_{0}+\left(\sigma_{m}+k^{*}\right) h\right)}{\varphi\left(t_{0}+\left(\sigma_{m}+k^{*}\right) h\right)} \leq \frac{K_{2}}{\sigma_{m}}
$$

and

$$
\left|z_{n^{\star}}\right| \leq B_{m}\left(1+\frac{K_{2}}{\sigma_{m}}\right)
$$

where $K_{2}$ is a positive real constant.

(iii) Let $R_{n^{*}-1} \neq 0$ and $R_{k}=0$ for some $k \in\left[\sigma_{m}, n^{\star}-2\right] \cap \mathbb{Z}^{+}$. This case is fully covered by the corresponding part of the proof of Theorem 3.2 .

The cases (i)-(iii) imply that

$$
\left|z_{n^{\star}}\right| \leq B_{m}\left(1+O\left(\frac{1}{\sigma_{m}}\right)\right) \quad \text { as } m \rightarrow \infty,
$$

where $n^{*} \in I_{m+1}$ and $n^{*}>\sigma_{m}$ is arbitrary. Hence $B_{m+1} \leq B_{m}\left(1+O\left(1 / \sigma_{m}\right)\right)$ and it remains to show that the product $\prod_{j=1}^{m}\left(1+1 / \sigma_{j}\right)$ converges as $m \rightarrow \infty$. Using the property $\delta \varphi\left(t+t_{0}\right) \geq \varphi\left(\delta t+t_{0}\right), t \geq 0, \delta \geq 1$ following from the properties of 
$\varphi$ stated in Proposition 4.1 we can write

$$
\begin{aligned}
\sigma_{m+1} & \geq \frac{1}{h}\left(\theta^{-1}\left(\left(\sigma_{m}-1\right) h+t_{0}\right)-t_{0}-h\right) \\
& =\frac{1}{h}\left(\varphi^{-1}\left(\frac{1}{\kappa} \varphi\left(\left(\sigma_{m}-1\right) h+t_{0}\right)\right)-t_{0}-h\right) \\
& \geq \frac{1}{h}\left(\varphi^{-1}\left(\varphi\left(\frac{1}{\kappa}\left(\sigma_{m}-1\right) h+t_{0}\right)\right)-t_{0}-h\right) \\
& =\frac{1}{\kappa} \sigma_{m}-\frac{1}{\kappa}-1 ;
\end{aligned}
$$

hence $\sigma_{m} \geq \kappa^{-m}\left(\sigma_{0}-\frac{1+\kappa}{1-\kappa}\right)$ and the corresponding infinite product converges. Now the validity of (4.7) follows from the boundedness of $B_{m}$ as $m \rightarrow \infty$.

We can verify that Theorem 4.4 actually represents the direct generalization of Theorem 3.2. Indeed, if $\theta(t)=\lambda t, 0<\lambda<1, t \geq 0$, then all the assumptions of Proposition 4.1 are satisfied and the corresponding Schröder equation

$$
\varphi(\lambda t)=\lambda \varphi(t), \quad t \geq 0
$$

admits the identity function as the required solution. Now obviously the asymptotic property (4.7) becomes (3.5).

To illustrate the applicability of Theorem 4.4 also to other types of delays we consider the differential equation (4.2) with the power delayed argument in the form

$$
y^{\prime}(t)=a(t) y(t)+b(t) y\left(t^{\omega}\right), \quad t \geq 1,
$$

where $0<\omega<1$ is a real scalar and $a, b$ are nonzero continuous functions on $[1, \infty)$. The trapezoidal formula (4.1) now yields the recurrence relation

$$
y_{n+1}=R_{n} y_{n}+S_{n} h\left(\beta_{n} y_{\left\lfloor\frac{(1+n h)^{\omega}-1}{h}\right\rfloor}+\alpha_{n} y_{\left\lfloor\frac{(1+n h)^{\omega}-1}{h}\right\rfloor+1}\right),
$$

where $R_{n}, S_{n}$ are given by (2.2) with $a_{n}=a(1+n h), b_{n}=b(1+n h)$ and

$$
\begin{aligned}
\alpha_{n}= & \frac{1}{2 h \omega}\left[(1+n h+h)^{\omega}-(1+n h)^{\omega}\right] \\
& \times\left[(1+n h)^{1-\omega}\left(\frac{(1+n h)^{\omega}-1}{h}-\left\lfloor\frac{(1+n h)^{\omega}-1}{h}\right\rfloor\right)\right. \\
& \left.+\frac{b_{n+1}}{b_{n}}(1+n h+h)^{1-\omega}\left(\frac{(1+n h+h)^{\omega}-1}{h}-\left\lfloor\frac{(1+n h)^{\omega}-1}{h}\right\rfloor\right)\right], \\
\beta_{n}= & \frac{1}{2 h \omega}\left[(1+n h+h)^{\omega}-(1+n h)^{\omega}\right] \\
& \times\left[(1+n h)^{1-\omega}+\frac{b_{n+1}}{b_{n}}(1+n h+h)^{1-\omega}\right]-\alpha_{n} .
\end{aligned}
$$

To apply the conclusion of Theorem 4.4 it is easy to check that the assumptions imposed on $\theta$ in Proposition 4.1 are satisfied. Then the asymptotic property (4.7) yields the effective result for the equation (4.9) provided we are able to solve explicitly the corresponding Schröder equation (4.4). This task is not difficult because considering $\theta(t)=t^{\omega}$ the relation (4.4) becomes the functional equation

$$
\varphi\left(t^{\omega}\right)=\omega \varphi(t), \quad t \geq 1
$$

with the solution $\varphi(t)=\log t$. Hence, we can present the following consequence of Theorem 4.4 . 
Corollary 4.5. Let $y_{n}$ be a solution of (4.9), where we assume the validity of the hypothesis (3.2) and let $\tilde{\gamma}$ be given by (3.3). Then

$$
y_{n}=O\left((\log n)^{-\log _{\omega} \tilde{\gamma}}\right) \quad \text { as } n \rightarrow \infty .
$$

Discussing some particular cases of (4.8) we can again observe close similarities between the formula (4.10) and the asymptotics of the exact equation (4.8) investigated, e.g. in [5. Indeed, it follows from Theorem 3.1 and Corollary 3.6 of [5] that under some additional assumptions on the coefficients $a$ and $b$, the upper bound for the exact solution of (4.8) can be expressed via the function $(\log t)^{-\delta}, \delta=\log _{\omega} Q$, where $Q>0$ is a majorant constant of the ratio $|b(t) / a(t)|$ which is assumed to be uniformly bounded on $[1, \infty)$. For other results discussing this type of asymptotics of the differential equations with a power deviating argument we refer to paper 22 ] (the delayed case) and to [7] (the advanced case).

These considerations warrant our posing the conjecture that the asymptotic property

$$
y_{n}=O\left(\varrho_{n}\right) \quad \text { as } n \rightarrow \infty,
$$

where $\varrho_{n}$ is a solution of (4.3) with the properties mentioned in Proposition 4.1 might be the "best" upper bound of the solutions $y_{n}$ of (4.1). The discussion and the precision of this conjecture will be the subject of future research.

The investigation of the common asymptotic properties of differential equations and their numerical discretizations is closely related to discussions on the unification of the differential and difference calculus. Recently, these discussions resulted in the origin and development of the theory of time scales (see [2]), which, among others, provide the flexible framework for the joint investigation of the qualitative properties of differential equations and their discretizations. Some basic results concerning delay equations are the subject of the paper [4, where the simplest (Euler) discretization of these equations has been considered.

\section{ACKNOWLEDGMENT}

The authors are grateful to an anonymous referee for numerous suggestions and comments which considerably helped to improve the content of this paper.

\section{REFERENCES}

1. A. Bellen and M. Zennaro, Numerical Methods for Delay Differential Equations, Numerical Mathematics and Scientific Computation, The Clarendon Press, Oxford University Press, New York, 2003. MR1997488(2004i:65001)

2. M. Bohner and A. Peterson, Dynamic Equations on Time Scales. An Introduction with Applications, Birkhäuser, Boston, 2001. MR 1843232 (2002c:34002)

3. M.D. Buhmann and A. Iserles, Stability of the discretized pantograph differential equation, Math. Comp. 60 (1993), 575-589. MR.1176707 (93h:65107)

4. J. Čermák, P. Kundrát and M. Urbánek, Delay equations on time scales: Essentials and asymptotics of the solutions, J. Difference Equ. Appl., 14(6) (2008), 567-580. MR2417009

5. J. Čermák, The asymptotic of solutions for a class of delay differential equations, Rocky Mountain J. Math. 33 (2003), 775-786. MR2038523 (2004j:34178)

6. M.L. Heard, A family of solutions of the IVP for the equation $x^{\prime}(t)=a x(\lambda t), \lambda>1$, Aequationes Math. 9 (1973), 273-280. MR0326105 (48:4450)

7. M.L. Heard, Asymptotic behavior of solutions of the functional differential equation $x^{\prime}(t)=$ $a x(t)+b x\left(t^{\alpha}\right), \alpha>1$, J. Math. Anal. Appl. 44 (1973), 745-757. MR0333405(48:11730)

8. M.L. Heard, A change of variables for functional differential equations, J. Differential Equations 18 (1975), 1-10. MR0387766(52:8605) 
9. C. Huang and S. Vandewalle, Discretized stability and error growth of the nonautonomous pantograph equation, SIAM J. Numer. Anal. 42 (2005), 2020-2042. MR2139235(2006c:65063)

10. A. Iserles, Numerical analysis of delay differential equations with variable delays, Ann. Numer. Math. 1 (1994), 133-152. MR.1340650 (96d:65130)

11. A. Iserles, On the generalized pantograph functional-differential equation, European J. Appl. Math. 4 (1993), 1-38. MR:1208418 (94f:34127)

12. A. Iserles, Exact and discretized stability of the pantograph equation, Appl. Numer. Math. 24 (1997), 295-308. MR 1464730 (98j:34130)

13. T. Kato and J.B. McLeod, The functional-differential equation $y^{\prime}(x)=a y(\lambda x)+b y(x)$, Bull. Amer. Math. Soc. 77 (1971), 891-937. MR0283338(44:570)

14. T. Kato, Asymptotic behaviour of solutions of the functional-differential equations $y^{\prime}(t)=$ $a y(\lambda t)+b y(t)$, in: K. Schmitt, Ed., Delay and Functional Differential Equations and Their Applications, Academic Press, New York, 1972, 197-218. MR0390432 (52:11258)

15. T. Koto, Stability of Runge-Kutta methods for the generalized pantograph equation, Numer. Math. 84 (1999), 870-884. MR.1730016 (2000k:65115)

16. M. Kuczma, B. Choczewski and R. Ger, Iterative Functional Equations, Encyclopedia Math. Appl., Cambridge Univ. Press, Cambridge, 1990. MR.1067720 (92f:39002)

17. H. Lehninger and Y. Liu, The functional-differential equation $y^{\prime}(t)=A y(t)+B y(\lambda t)+$ $C y^{\prime}(q t)+f(t)$, European J. Appl. Math. 9 (1998), 81-91. MR1617009 (99i:34089)

18. Y. Liu, Asymptotic behaviour of functional-differential equations with proportional time delays, European J. Appl. Math. 7 (1996), 11-30. MR1381795 (97c:34164)

19. Y. Liu, On the $\theta$-method for delay differential equations with infinite lag, J. Comput. Appl. Math. 71 (1996), 177-190. MR1399890 (97c:65136)

20. Y. Liu, Numerical investigation of the pantograph equation, Appl. Numer. Math. 24 (1997), 309-317. MR:1464731 (98j:34131)

21. M. Z. Liu, Z. W. Yang and Y. Xu, The stability of the modified Runge-Kutta methods for the pantograph equation, Math. Comp. 75 (2006), 1201-1215. MR2219025 (2007b:65061)

22. G. Makay and J. Terjéki, On the asymptotic behavior of the pantograph equations, Electron. J. Qual. Theory Differ. Equ. 2 (1998), 1-12. MR.1615106 (99a:34215)

23. M. Sezer and A. Dascioğlu, A Taylor method for numerical solution of generalized pantograph equations with linear functional argument, J. Comput. Appl. Math. 200 (2007), 217-225. MR:2276827(2007k:65100)

Institute of Mathematics, Brno University of Technology, Technická 2, CZ-616 69 Brno, Czech Republic

E-mail address: cermak.j@fme.vutbr.cz

Institute of Mathematics, Brno University of Technology, Technická 2, CZ-616 69 Brno, Czech Republic

E-mail address: yjansk04@stud.fme.vutbr.cz 